\title{
The JPEG image query feedback research based on
}

\section{the Compressed domain}

\author{
Jianfeng Wang ${ }^{1}$ Zikun Liu' $\quad$ Mingke $\mathrm{Li}^{1}$ Wenming $\mathrm{Wu}^{1} \quad$ Xiaorong Zhao ${ }^{1}$
}

(1,ChongQing aerospace polytechnic,400021,Chongqing)

Key words: DCT , CBIR, Compressed domain , Feedback

\begin{abstract}
The research focus of the CBIR is to improving the accuracy of the image retrieval with the feedback technology. But this technology can not solve the problem between the low-level feature and high-level semantic, which is the difficulty of the CBIR and the difficulty of the image compressing and video retrieval. This paper shows the basic method of image retrieval in the compressed domain and the developing situation of the feedback. One complicated feedback framework at the CBIR are proposed.
\end{abstract}

\section{Introduction}

In recent years, with the rapid development of multimedia technology and computer network, the digital image of the world is growing at an alarming rate. Whether it is military or civilian equipment, every day will produce a capacity equivalent to thousands of images of a byte, these digital images contain a lot of useful information. However, since these images are disordered distribution in all parts of the world, the information contained in the image can not be effectively accessed and utilized. This requires that there is a technique that can quickly and accurately find access to the image, which is called the content-based image retrieval technique (CBIR). CBIR attempts to automate the indexing process in an image database. CBIR method is based on the image intrinsic properties such as color, texture, shape and statistical characteristics of the description and retrieval work ${ }^{[1][2][3][4]}$. Still, the retrieval accuracy of the CBIR algorithm is still limited. In addition to many other difficulties, the bottleneck of CBIR is the gap between low-level visual features and high-level semantic features. The source of the bottleneck lies in the fact that the visual similarity measure, such as the color histogram, and so on, is not necessarily the semantic matching of the image in the human subjective definition. In order to solve the bottleneck, the interactive relevance feedback technology is proposed. The central idea is we should have the opportunity to understand human subjectivity into the image retrieval process and give users to evaluate the retrieval results, in the assessment of user based on further improved the retrieval process.

\section{Classical feedback algorithm}

The relevance feedback scheme of early CBIR mainly comes from the classical text documents. The classical methods are divided into two categories: query point movement (the query point 
movement, query refinement) and then weighted (re-weighting, similarity measure refinement) [5], the two methods are based on vector model development, and model vector is the most popular model in information retrieval.

Query point moving method is essentially by making the "ideal query point" move to the good sample point and to improve the evaluation value from the bad sample point. The often used technique to improve the estimation is Rocchio (1), which is a collection of DR and DN, a collection of relevant documents given by the user:

$$
Q=\alpha Q+\beta\left(\frac{1}{N_{R^{\prime}}} \sum_{i \in D_{R}} D_{i}\right)-\gamma\left(\frac{1}{N_{N^{\prime}}} \sum_{i \in D_{N}} D_{i}\right)
$$

Re weighting method and the basic idea is to improve those help to retrieve the weights of the features of the image correlation scale, while reducing the hindrance to the weights of the features of the image correlation scale retrieval. The MARS system has realized the small improvement of the re weighting method which is called the standard deviation method [6]

\section{Proposed relevance feedback algorithm}

\section{With memory and no memory feedback}

One drawback of the classical relevance feedback method is that the knowledge acquired during the retrieval of the relevance feedback is not stored in order to improve the retrieval performance. That is to say, even for the same query, a user usually has to get the same results through the same tedious feedback process, even though the fact that the user has previously given the same retrieval requirements and feedback.

The basic idea is to improve the performance of CBIR by using the information of the user in the related network. In mathematics, the relevant network is described by a matrix $\mathrm{M}$, which is defined as follows ( 2 ):

$$
M=\left[\begin{array}{rrrr}
w_{11} & w_{12} & \mathrm{~L} & w_{1 N} \\
w_{21} & w_{22} & \mathrm{~L} & w_{2 N} \\
\mathrm{M} & & & \mathrm{M} \\
w_{N 1} & w_{N 2} & \mathrm{~L} & w_{N N}
\end{array}\right]
$$

Which, the matrix elements (known as weight or coefficient), the semantic correlation between the $i$ and the image cluster $j$.

Firstly, all images in the database are based on visual features (such as: using K- means) are divided into $\mathrm{N}$ group. It is obvious that the image in each group is similar, which is similar to the classical CBIR system. Moreover, the correlation coefficient between the first two groups was set to 0 , which is not related to the two groups. And any group is entirely related to itself. Namely: the initial matrix is a unit matrix, as described by the formula (3):

$$
M_{0}=I_{N \times N}
$$

Then, to a given query request, the retrieval is based on visual features. After a repeated process, the $n+m$ images are displayed as a result, the $n$ images are labeled as the correlation, and the other $\mathrm{m}$ images are not related. Whether it is relevant or not relevant, it may come from the same group, 
and it may come from different groups. The system is updated by the type (4) to update the related matrix to the memory:

$$
M_{t}=M_{t-1}+\sum_{i=1}^{m} F(q) F\left(p_{i}\right)^{T}-\sum_{i=1}^{n} F(q) F\left(n_{i}\right)^{T}
$$

Which $\mathrm{q}$ is the query feature vector, pi and ni is a character vector of the feedback of positive and negative examples, $\mathrm{F}(\mathrm{x})$ is a conversion function, used to determine sample update rate based on feedback. In this way, the correlation between the original group and the normal group was enhanced. The correlation is used to retrieve the similarity between the visual features and the semantic relevance, which can be used to determine the similarity of the images. The advanced learning method is proved to be effective in the use of the previous information.

\section{Feedback network}

Considering the above factors, we propose a framework for content-based image retrieval in the DCT domain, which integrates relevant feedback and query expansion. In this framework, the integration of semantic and underlying feature vector based indexing and relevance feedback is integrated. Figure 4 describes the framework, which includes semantic web based on the database, the semantic web, the semantic features of the semantic features and the image features, and a machine learning algorithm, which is updated with time. This system is supported by the semantic web and the underlying feature index, which supports the query and the image sample. More importantly, the learning process in the feedback process can be the key words annotation from the annotated image to the unlabeled images, so that through the semantic transmission process, more and more images are secretly marked on the notes. This annotation process also helps to accumulate knowledge and improve the retrieval performance.
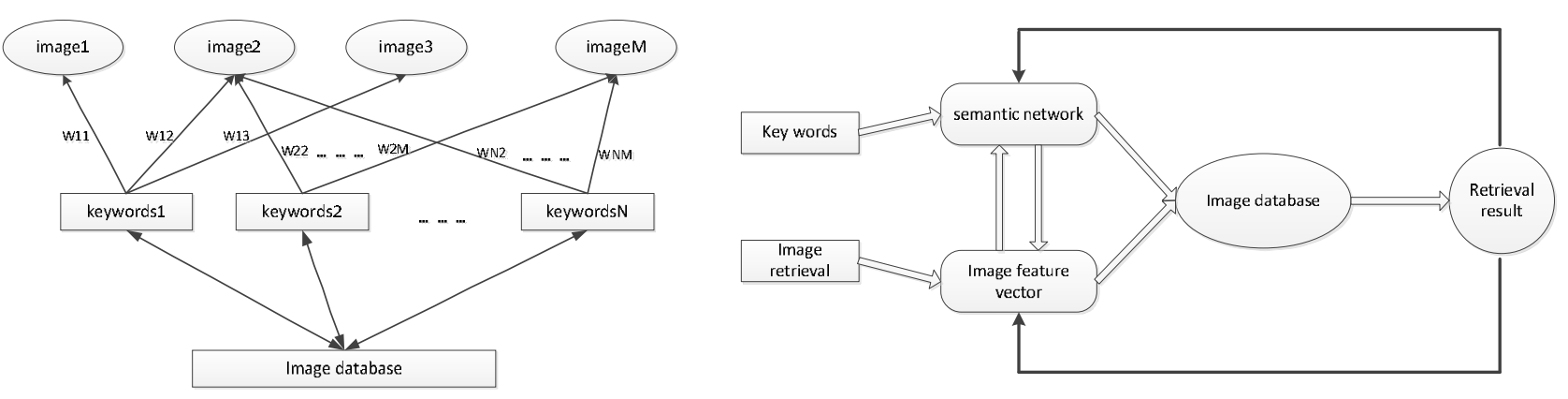

Among them, the semantic web is a two layer structure, as shown in figure 1, 2, the top is a library of images, each keyword connected to correspond to the image in the image library. Keywords associated with the image of correlation between the semantic content of each by the picture on the connection weights. This layer is used for keywords related to feedback and updated in the process of semantic transmission. The bottom is a keyword dictionary, is used to establish the connection between the different keywords

\section{Algorithm Evaluations via Experiments}

Using MATLAB to achieve the performance of the proposed algorithm, the image database consisting of 3000 images is set up, including landscape, flowers, face, vehicle four kinds of images and some interference images. For comparison, this paper on the above experimental data were used benchmark ${ }^{[1-2]}$ and proposed method respectively for content-based image retrieval (CBIR) test, using precision, recall to evaluate retrieval performance of the proposed algorithm, In order to 
quantify the performance of the algorithm, the comprehensive evaluation index $\mathrm{F}$ is defined (5).

$$
F=2 \times \frac{P \times R}{P+R}
$$

The experimental results are shown in Table-I .From these experimental results, it can be seen that: the retrieval precision of the proposed algorithm is overwhelmingly better than the benchmark in terms of both $\mathrm{p}$ and $\mathrm{r}$

Table-I Experimental Results for Retrieving performance

\begin{tabular}{|c|c|c|c|c|c|c|c|c|c|}
\hline \multirow{2}{*}{ image } & \multicolumn{3}{|c|}{ our algorithm } & \multicolumn{3}{c|}{ Benchmark[1] } & \multicolumn{3}{c|}{ Benchmark[2] } \\
\cline { 2 - 11 } & $P$ & $R$ & $F$ & $P$ & $R$ & $F$ & $P$ & $R$ & $F$ \\
\hline landscape & 0.85 & 0.87 & 0.86 & 0.66 & 0.62 & 0.64 & 0.59 & 0.81 & 0.68 \\
\hline flowers & 0.77 & 0.79 & 0.78 & 0.67 & 0.58 & 0.62 & 0.64 & 0.65 & 0.64 \\
\hline face & 0.78 & 0.85 & 0.81 & 0.74 & 0.71 & 0.72 & 0.72 & 0.63 & 0.67 \\
\hline vehicle & 0.81 & 0.87 & 0.84 & 0.70 & 0.79 & 0.74 & 0.74 & 0.61 & 0.67 \\
\hline
\end{tabular}

\section{Summary}

Through the analysis of this paper, the most critical problems in CBIR system are as follows: 1 how to focus on effective learning from the feedback samples, 2) how to accumulate knowledge from feedback, 3) to integrate the underlying visual features and high-level semantic features in the retrieval and feedback. This paper presents an effective solution to these problems. Through feedback can improve the accuracy of image retrieval, can establish and user interface, and in the DCT domain without all decoding, thus greatly improve the retrieval speed, which is also used in video retrieval.

\section{ACKNOWLEDGEMENT}

Science and technology project of Chongqing Municipal Education Commission (item number: kj133201)

Science and technology project of Chongqing Municipal Education Commission (item number: kj133203)

\section{Reference}

[1]Hermes $\mathrm{T}$.et a1 .. Image retrieval for information systems in storage and retrieval for image and video Databases III . In : Proceedings of SPIE 2420 . San Jose , CA , 2005

[2]Beitao Li Edward Chang, Ching-Tung Wu,DPF-A Perceptual Distance Function for Image Retrieval. No.6,2006

[3] Editorial, Content-Based Image and Video Retrieval, Signal Processing 85 (2005) 231-232 
[4] NUNO VASCONCELOS, ANDREW LIPPMAN, A Multiresolution Manifold Distance for Invariant Image Similarity, IEEE TRANSACTIONS ON MULTIMEDIA,VOL.7, NO.1,FEBRUARY 2005

[5] Buckley, C., and Salton, G. "Optimization of Relevance Feedback Weights," in Proc of SIGIR'95.

[6] Minka, T. and Picard, R. "Interactive Learning using a 'Society of Models', Pattern Recognition, 30(4),2007. 